\title{
Extraction and purification of phenolic compounds from lignocellulosic biomass assisted by ionic liquid, polymeric resins and supercritical $\mathrm{CO}_{2}$
}

André M. da Costa Lopes, ${ }^{1,2}$ Miriam Brenner, ${ }^{1}$ Pedro Falé, ${ }^{3}$ Luísa B. Roseiro, ${ }^{1}$ Rafał Bogel-Łukasik ${ }^{1 *}$

1 Laboratório Nacional de Energia e Geologia, Unidade de Bioenergia, 1649-038 Lisboa, Portugal.

2 LAQV/REQUIMTE, Departamento de Química, Faculdade de Ciências e Tecnologia, Universidade Nova de Lisboa, 2829-516 Caparica, Portugal.

${ }^{3}$ Centro de Química e Bioquímica. Faculdade de Ciências, Universidade de Lisboa, Campo Grande, 1749-016 Lisboa, Portugal.

* Corresponding author: fax: +351217163636; phone: +351210924600 ext. 4224; email address: rafal.lukasik@lneg.pt 
Table S1. Doehlert experimental design applied for the corresponding experimental responses $Y_{1}$ (total phenolic extraction yield) and $Y_{2}$ (vanillin extraction yield).

\begin{tabular}{lllll}
\hline \multirow{2}{*}{ Entry } & \multicolumn{3}{l}{ Coded variables } & \multicolumn{2}{l}{ Responses } \\
\cline { 2 - 5 } & $X_{1}$ & $X_{2}$ & $Y_{1}(\%)$ & $Y_{2}\left(\mu \mathrm{g} / \mathrm{g}_{\text {biomass }}\right)$ \\
\hline A & -0.03 & -0.40 & 74 & 456 \\
B & 0.83 & -0.40 & 73 & 502 \\
C & -0.90 & -0.40 & 50 & 292 \\
D & 0.40 & 0.12 & 79 & 485 \\
E & -0.47 & 0.92 & 54 & 386 \\
F & 0.40 & 0.92 & 73 & 488 \\
G & -0.47 & 0.12 & 66 & 482 \\
H & -0.90 & 0.80 & 43 & 265 \\
\hline
\end{tabular}

The statistical significance of estimated effects on both $Y_{1}$ and $Y_{2}$ responses was checked by analysis of variance (ANOVA) presented in Table 2. The $p$-values indicated the statistical significance $(p<0.05)$ of the estimated relations between variables within a $95 \%$ confidence interval for obtained coefficients.

Table S2. Parameters of the polynomial models representing the studied response $Y_{1}$ (total phenolic extraction yield) and $Y_{2}$ (vanillin extraction yield); The adequacy of the models to fit the sets of data was performed using Fisher test (F-test) for the effectiveness of the factors .

\begin{tabular}{ccccc}
\hline \multirow{2}{*}{ Model parameters (MP) } & \multicolumn{3}{c}{$Y_{1}$} & \multicolumn{2}{c}{$Y_{2}$} \\
\cline { 2 - 6 } & $M P$ & $p$ & $M P$ & $p$ \\
\hline$b_{0}$ & 76.54 & 0.00 & 497.65 & 0.00 \\
$b_{1}$ & 11.27 & 0.00 & 73.00 & 0.04 \\
$b_{2}$ & -3.70 & 0.28 & -42.92 & 0.34 \\
$b_{12}$ & -6.24 & 0.20 & -58.60 & 0.36 \\
$b_{11}$ & -18.24 & 0.00 & -131.40 & 0.01 \\
$b_{22}$ & -15.37 & 0.00 & -92.97 & 0.11 \\
\hline \multicolumn{5}{c}{ F-test } \\
Effectiveness of the parameters & 48.66 & & & \\
Significance level (p<0.05) & 0.00 & 0.00 \\
\hline $\mathrm{R}^{2}$ & 0.96 & \multicolumn{2}{c}{0.87} \\
\hline
\end{tabular}




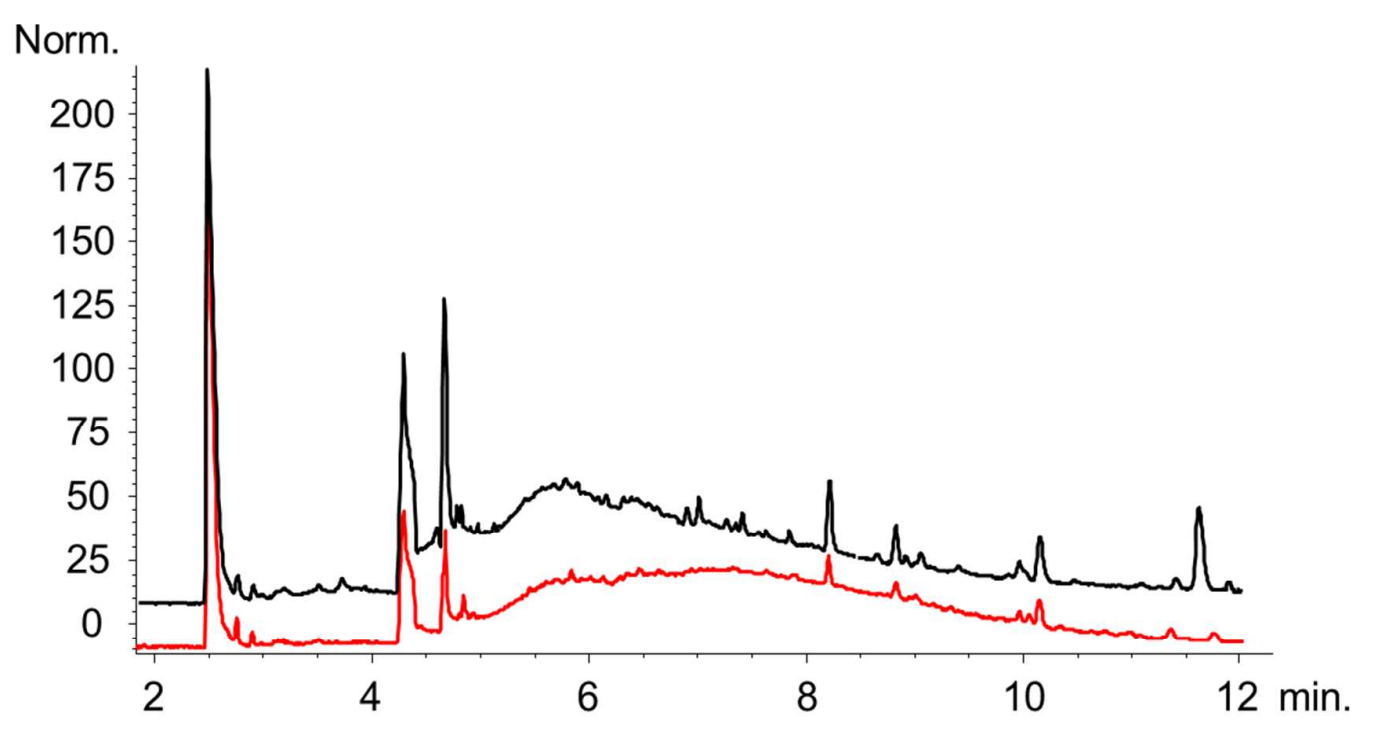

Figure S1. Phenolic adsorption profiles of the original sample placed on the pressure vessel before (black) and after (red) $\mathrm{CO}_{2}$ extraction, both recorded at $200 \mathrm{~nm}$ wavelength. See text for CE phenolics separation conditions. 